\title{
The drop height determines neuromuscular adaptations and changes in jump performance in stretch-shortening cycle training
}

\author{
W. Taube ${ }^{1}$, C. Leukel ${ }^{1,2}$, B. Lauber ${ }^{2}$, A. Gollhofer ${ }^{2}$ \\ ${ }^{1}$ Department of Medicine, Unit of Sport Science, University of Fribourg, Fribourg, Switzerland, ${ }^{2}$ Department of Sport Science, \\ University of Freiburg, Fribourg, Germany \\ Corresponding author: Wolfgang Taube, Department of Medicine, Unit of Sport Science, University of Fribourg, 1700 \\ Fribourg, Switzerland. Tel: +4126300 7283, Fax: +41263009771, E-mail: wolfgang.taube@unifr.ch
}

\begin{abstract}
There is an ongoing discussion about how to improve jump performance most efficiently with plyometric training. It has been proposed that drop height influences the outcome, although longitudinal studies are missing. Based on crosssectional drop jump studies showing height-dependent Hoffmann (H)-reflex activities, we hypothesized that the drop height should influence the neuromuscular activity and thus, the training result. Thirty-three subjects participated as a control or in one of two stretch-shortening cycle (SSC) interventions. Subjects either trained for 4 weeks doing drop jumps from 30, 50, and $75 \mathrm{~cm}$ drop heights (SSC1) or completed the same amount of jumps exclusively from $30 \mathrm{~cm}$ (SSC2). During training and testing (from 30, 50, and $75 \mathrm{~cm}$ ), subjects were instructed to minimize the duration of ground contact and to maximize their rebound height. Rebound heights were significantly augmented after
\end{abstract}

In many sports disciplines, jump performance in general, and vertical jumping height and duration of ground contact time in particular, is considered as key qualifications. To improve jump performance, training interventions including stretch-shortening cycle (SSC) movements (Markovic, 2007) are regarded as being superior to exercise protocols like heavy and explosive resistance training (Wilson et al., 1993, 1996), electrostimulation (Malatesta et al., 2003) or vibration training (Cardinale \& Bosco, 2003), which do not involve SSC contractions. Nevertheless, there exists a great variation in the outcome of SSC training protocols (Markovic, 2007). Part of this variation is thought to be due to differences in the jumping technique and the drop height applied during training (Bobbert et al., 1987a, 1987b; Bobbert, 1990), although longitudinal studies addressing this issue are missing. Furthermore, jumping technique (e.g. the extent of countermovement, time of ground contact, muscular activation pattern) and drop height were most often separately treated (Bobbert et al., 1987a, 1987b; Hunter \& Marshall, 2002), even though cross-sectional studies have demonstrated that the drop height influences
SSC1, but a trend was only observed after SSC2. In contrast, the duration of ground contact increased after SSC1 but decreased after SSC2. The performance index (rebound height/duration of ground contact) improved similarly after SSC1 $(+14 \%)$ and SSC2 $(+14 \%)$. Changes in performance were accompanied by neuromuscular adaptations: for SSC1, activity of the soleus increased toward take-off (between 120 and $170 \mathrm{~ms}$ after touchdown), whereas SSC2-trained subjects showed enhanced activity shortly after ground contact (20-70 ms after touch down). The present study demonstrates a strong link among drop height, neuromuscular adaptation, and performance in SSC training. As the improvement in the performance index was no different after SSC1 or SSC2, the decision whether to apply SSC1 or SSC2 should depend on the specific requirements of the sports discipline.

the neuromuscular activity and thus, the jumping technique (Komi \& Gollhofer, 1997; Leukel et al., 2008a, 2008b). These cross-sectional studies further highlighted that the muscular activity shortly after ground contact at the time of the short latency response (SLR) is particularly influenced by the drop height. When subjects were asked to perform drop jumps from different drop heights, the muscular activity at the SLR was lower in drop jumps from greater heights $(80 \mathrm{~cm})$ than from low heights $(30 \mathrm{~cm})$ (Komi \& Gollhofer, 1997). In a subsequent experiment, the size of the Hoffmann (H)-reflex was found to be inversely related to the drop height (Leukel et al., 2008a, 2008b). The decreased muscular activity and H-reflex excitability at greater heights were argued to be caused by a prevention strategy in order to reduce stress on the tendo-muscular system in high-load conditions.

Recently, Zuur et al. (2010) confirmed the longheld assumption of stretch-induced reflex activity at the time of the SLR in hopping. The authors altered the time of ground contact by changing the height of the floor surface, while subjects were airborne and unaware of this procedure. The latency of the SLR 
was exactly time-locked to the instant of ground contact resulting in prolonged latencies in the condition where the landing surface was lowered (due to the longer air time) and in shortened latencies where the landing surface was raised. In SSC movements, these stretch reflex contributions are considered to be an important factor in the ability to transfer energy from the pre-activated and eccentrically stretched muscle-tendon complex to the concentric push-off phase (Dietz et al., 1979; Gollhofer et al., 1992; Voigt et al., 1998b).

As spinal stretch reflexes and H-reflexes are dependent on the drop height (Komi \& Gollhofer, 1997; Leukel et al., 2008a) and reflexes influence the stiffness characteristics of the tendo-muscular complex (Hoffer \& Andreassen, 1981; Hobara et al., 2007), it was hypothesized that training from different drop heights should induce specific neuromuscular adaptations, which in turn influence the jumping technique; more specifically, the resulting muscular activity at the SLR during drop jumps from low heights $(30 \mathrm{~cm}$; Komi \& Gollhofer, 1997) was hypothesized to result in a higher muscular stiffness and therefore an improved capacity to store elastic energy when this pattern is reinforced by exercise. This increased muscular stiffness should also enable subjects to minimize their time of ground contact. On the other hand, SSC training incorporating high drop heights was expected to induce adaptations in the concentric rather than the eccentric phase due to the previously observed load-dependent electromyogram (EMG) and H-reflex inhibition at the SLR. Emphasis on the concentric action was assumed to prolong the ground contact times due to an expected reduction in muscular stiffness. The aim of the present study was to identify the influence of the drop height on jump performance by testing the aforementioned hypotheses. For this purpose, two plyometric training protocols were applied; in SSC1, subjects performed drop jumps from high and low drop heights $(30,50,75 \mathrm{~cm})$, whereas the SSC2 training group jumped solely from a low height $(30 \mathrm{~cm})$. Neurophysiological (surface EMG, H-reflexes) and biomechanical parameters (kinematic data, ground reaction force, and rebound height) were used to indicate training-related changes. The relationship between muscular activity and muscular stiffness could not be directly assessed as the length of the muscle fascicles was not monitored. However, before and after training, the ankle joint stiffness was calculated, which is considered to be strongly dependent on the muscular stiffness of the triceps surae.

\section{Materials and methods}

Subjects

Thirty-three healthy subjects with no history of neurological disorders or injuries to the lower extremities volunteered to participate in this study. All subjects participated in sporting activities containing jumps (volleyball, basketball, athletics, etc.) to minimize variations in jumping performance and guarantee high fatigue resistance during the measurements. Thirty-three subjects were randomly allocated into a SSC group training from low $(\mathrm{LH} ; 30 \mathrm{~cm})$, moderate $(\mathrm{MH}$; $50 \mathrm{~cm})$ and high $(\mathrm{HH} ; 75 \mathrm{~cm})$ heights $(\mathrm{SSC} 1$; four females and seven males; $24 \pm 3$ years, weight: $68 \pm 11 \mathrm{~kg}$, height: $177 \pm 9 \mathrm{~cm}$ ), a second training group training exclusively from LH (SSC2; four females and seven males; $25 \pm 4$ years, weight: $69 \pm 8 \mathrm{~kg}$, height: $179 \pm 9 \mathrm{~cm}$ ) and a control group $(\mathrm{CON}$; six females and five males; $24 \pm 3$ years, weight: $63 \pm 9 \mathrm{~kg}$, height: $173 \pm 7 \mathrm{~cm}$ ). None of the subjects participated in any other systematic training during the experiment or had performed previously a similar SSC training. All subjects gave written informed consent to the experimental procedure, which was in accordance with the Declaration of Helsinki and approved by the ethics committee of the AlbertLudwigs-University in Freiburg.

\section{SSC1 and SSC2 training}

SSC training was performed three times per week for 4 weeks (12 training sessions), which were all surveyed and supervised by the authors of the study. Each training session lasted for 45-60 min including a 10-min warm up (jogging, mobilization exercises for the trunk muscles and hopping). The SSC1 exercises consisted of drop jumps from three different heights: 30,50 , and $75 \mathrm{~cm}$. Twelve jumps were performed in each training session from 30 and $50 \mathrm{~cm}$, respectively. From the $75 \mathrm{~cm}$ height, the number of jumps was successively increased from six drop jumps in the first week to 8 (second week), 10 (third week), and finally 12 jumps (fourth week) to accustom the subjects to the strong impact. The sequence of the three dropping heights was randomized during the training. The SSC1 training was not exclusively restricted to high drop heights, as this would have put too much stress on the tendo-muscular system of the participating subjects. It was considered to be more important that the subjects performed the same amount of jumps in the two training regimes in order to compare both interventions. Furthermore, the amount of jumps was chosen to resemble a realistic training session. In the SSC2 group, subjects performed the same total amount of jumps as the SSC2 group but they jumped solely from LH. All subjects were instructed to perform highly concentrated jumps with maximal intensity, i.e. with minimum duration of ground contact and maximal rebound height. Subjects had to rest $10 \mathrm{~s}$ after each jump and $10 \mathrm{~min}$ after they completed all jumps from one drop height (SS1) or, in the SS2 group, after 12 (first round) and 24 jumps (second round) from the LH condition. The number of jumps in the last round in the SSC2 group was dependent on the number of jumps subjects of the SSC1 group performed at the $\mathrm{HH}$. Thus, the number of jumps was successively increased from 6 to 12 jumps. Subjects of the control group maintained their normal physical activities throughout the experimental period and were measured again 4 weeks after their initial test.

\section{General experimental procedure}

The experiments were conducted before and after the training period. The post-measurement occurred approximately $48 \mathrm{~h}$ after the last training session in order to avoid fatigue.

The testing procedure resembled previously reported ones (Leukel et al., 2008a; Taube et al., 2008) and consisted of a 5min warm-up (low height hopping) to accustom the subjects to the jumping procedure. Before each jump, both hands had to be held akimbo (hands at the ilium), the left leg remained 
stable in order to secure an upright stance whereas the right leg was lifted and projected in front of the body. The jump off was performed with the left leg to keep the right leg (from which the muscular activity was recorded) relaxed until jump off. Subjects were asked to touch down on both feet and to jump off the ground as quickly as possible. Subjects were also instructed to keep this jumping procedure similar throughout the whole experiment. Drop jumps were performed from three different falling heights: low $(\mathrm{LH}, 30 \mathrm{~cm})$, moderate $(\mathrm{MH}$, $50 \mathrm{~cm})$, and high $(\mathrm{HH}, 75 \mathrm{~cm})$. From each drop height $(30,50$, and $75 \mathrm{~cm}$ ), five jumps with maximal intensity were performed to measure the maximal individual jump height. Additionally, 10 jumps at submaximal intensity without peripheral nerve stimulation were recorded to assess activity in the soleus muscle (SOL) during the drop jump. Based on these jumps, the temporal occurrence of the peak of the SLR was determined in the rectified and averaged EMG. Peripheral nerve stimulation was timed so that the peak of the H-reflex coincided with either the instant of ground contact (GND) or the peak of the SLR (in line with Leukel et al., 2008a, 2008b). H-reflex excitability at GND was assessed as a reference to SLR. Stimulation at GND was triggered by means of a light barrier mounted $25 \mathrm{~cm}$ above the ground for the $\mathrm{LH}$ condition and $35 \mathrm{~cm}$ for the $\mathrm{MH}$ and $\mathrm{HH}$ conditions. Depending on the individual latencies of the $\mathrm{H}$ reflex and the time between passing the barrier and touch down on a force plate (Kistler ${ }^{\circledR}$, Winterthur, Switzerland), electrical stimulation was task-specifically triggered so that the $\mathrm{H}$-reflex coincided with the moment of ground contact at each height (LH, MH, HH) (in line with Leukel et al., 2008a, 2008b). Subjects were instructed to rest for $2 \mathrm{~min}$ after every 20 jumps to avoid fatigue.

\section{EMG recording}

Muscle activity was recorded from the SOL, the gastrocnemius medialis (GM), the rectus femoris (RF), and the tibialis anterior (TA) muscles of the right leg. $\mathrm{Ag}-\mathrm{AgCl}$ bipolar surface electrodes (Hellige ${ }^{B}$, Freiburg, Germany, diameter $9 \mathrm{~mm}$, center-to-center distance $3 \mathrm{~cm}$ ) were filled with electrode jelly and attached to the skin over the muscle of interest in line with the presumed direction of the underlying muscle fibers. The reference electrode was placed on the tibial plateau. The position of the electrodes was marked with a waterproof felt pen and the markings were renewed in the training sessions from time to time. EMG signals were amplified $(\times 1000)$, bandpass-filtered $(10-1000 \mathrm{~Hz})$ and sampled at $4 \mathrm{kHz}$.

\section{Peripheral nerve stimulation}

Rectangular current pulses lasting $1 \mathrm{~ms}$ were delivered to the posterior tibial nerve by a constant current stimulator (AS100 Alea Solutions ${ }^{B}$, Zurich, Switzerland). The anode, a $10 \times 5 \mathrm{~cm}$ dispersal pad, was fixed on the anterior aspect of the knee just below the patella. The cathode $(2 \mathrm{~cm}$ in diameter) was placed in the popliteal fossa and moved stepwise until the best position for eliciting an H-reflex in the soleus muscle was found. It was ensured that electrical stimulation evoked no response in the TA muscle. The cathode was fixed with rigid tape. At the beginning of the experiment, $\mathrm{H}$-reflex recruitment curves were recorded during normal stance. As the tibial nerve contains both, sensory and motor axons, electrical stimulation of this nerve elicits a reflectory response (H-reflex with a latency of approximately $35 \mathrm{~ms}$ ) and a direct muscle response (M-wave; latency $\sim 10 \mathrm{~ms}$ ) in the SOL. The H-reflex is mediated via Ia afferents to the $\alpha$-motoneuron and from there on to the neuromuscular junction. The M-wave travels from the point of stimulation directly via motor axons to the neuromuscular junction. Low stimulation intensity results in a small $\mathrm{H}$-reflex without any $\mathrm{M}$-wave because the large diameter Ia afferents are recruited before the smaller motor axons. When stimulation intensity is successively increased from low levels sufficient to elicit a small $\mathrm{H}$-reflex, up to $H_{\max }$ and then $M_{\max }$, an H-reflex recruitment curve can be obtained (Zehr, 2002). Expressing the maximal $H$-reflex relative to $M_{\max }$ $\left(H_{\max } / M_{\max }\right.$ ratio) solves the potential problem of ensuring identical EMG recording conditions in pre- and post-training (Aagaard et al., 2002). In the present study, SOL H-reflex recruitment curves were obtained during drop jumps at GND and at the time of the SLR at each height ( $\mathrm{LH}, \mathrm{MH}, \mathrm{HH})$.

\section{Kinematics}

From each height, five jumps were recorded with a high-speed camera (100 frames per second) and analyzed afterwards using a commercially available video analysis system (Simi Motion ${ }^{B}$, Unterschleissheim, Germany). Anatomical landmarks (shoulder, hip, knee, and ankle center as well as the toe-cap) were marked to digitalize the subjects' movements and to obtain ankle, knee, and hip angles. The kinematic measurements were performed for SS1 and the control group by the high-speed camera and additionally by goniometers. The SSC2 group was measured in parallel and the joint angles in the SSC2 group were solely recorded using goniometers placed at the hip, the knee, and the ankle.

\section{Data analysis}

Based on the H-reflex recruitment curves, maximum H-reflex $\left(H_{\max }\right)$ and maximum $\mathrm{M}$-wave amplitudes $\left(M_{\max }\right)$ were determined. $H_{\max } / M_{\max }$ ratios $(H / M$ ratios) were calculated for each condition in the pre- and post-measurement and compared before and after training. Additionally, $H / M$ ratios recorded during jumping were expressed as a percentage of the reference value obtained standing at rest (analogous to Voigt et al., 1998a).

Muscular activity during drop jump was analyzed in four time periods: $t_{1}=100 \mathrm{~ms}$ before ground contact to the duration of ground contact ( -100 to $0 \mathrm{~ms}$; "pre-activation phase"); $t_{2}=20-70 \mathrm{~ms}$ after ground contact ("early reflex phase," which incorporated the SLR); $t_{3}=70-120 \mathrm{~ms}$ ("late reflex phase" incorporating long latency reflexes); and $t_{4}=120-170 \mathrm{~ms}$ ("push-off phase"). Root mean square values were determined for each phase of the drop jump. In the case of the SOL muscle, muscular activity was normalized to the $M_{\max }$ obtained in the same muscle. The jumping height in trials testing maximum jump height was calculated by the formula: jump height $=1 / 8 \times g \times t^{2}$, where $g$ is the acceleration due to gravity and $t$ is the time of the flight phase. For evaluating drop jump performance, jump height as well as the duration of ground contact were considered to be of relevance. Thus, a performance index was calculated as the relationship between jump height and the duration of ground contact (performance index $=$ height/time; Bruhn et al., 2004; Taube et al., 2007). Based on the kinematic data, the maximal countermovement (angular joint amplitudes) was determined for each subject and the corresponding angle joints of hip, knee, and ankle were compared before and after SSC training. Ankle joint stiffness was calculated for the eccentric phase of the drop jump according to Kubo et al. (2007). First, ankle joint torque (TQ) during the drop jump was estimated from the following equation (Kawakami et al., 2002; Kubo et al., 2007):

$$
T Q=F_{\mathrm{z}} L 1 \cos \left(A_{\mathrm{j}}-90\right)
$$


where $F_{\mathrm{z}}, \mathrm{L} 1$, and $A_{\mathrm{j}}$ are the vertical component of the ground reaction force, the length from the ankle joint to the ball of the foot, and the ankle joint angle, respectively. The ankle joint stiffness was calculated as the change in joint torque divided by the change in the ankle joint angle during the eccentric phase (Kuitunen et al., 2002).

\section{Statistics}

To identify potential differences between groups in the premeasurement, one-way ANOVAs were conducted for each parameter (rebound height, duration of ground contact, performance index, etc.). Changes in jumping height (rebound height), duration of ground contact, and the performance index were analyzed using a repeated measures ANOVA procedure with the within-subject factors time (pre- vs posttraining measurement) and drop height ( $\mathrm{LH}, \mathrm{MH}, \mathrm{HH})$ and the between-subject factor group (SSC1, SSC2 and CON) $[2$ $($ time $) \times 3$ (condition) $\times 3$ (group) $]$. A repeated-measures ANOVA with the factors time (pre, post), drop height ( $\mathrm{LH}, \mathrm{MH}$, $\mathrm{HH}$ ), time of stimulation (ground vs SLR), and group (SSC1, $\mathrm{SSC} 2, \mathrm{CON})[2$ (time) $\times 3$ (condition) $\times 2$ (time of stimulation) $\times 3$ (group)] was conducted to evaluate alterations in the $H / M$ ratios. Changes in the normalized muscular activity of the SOL were analyzed by using a repeatedmeasures ANOVA with the factors time (pre, post), drop height ( $\mathrm{LH}, \mathrm{MH}, \mathrm{HH})$, phase $\left(t_{1}, t_{2}, t_{3}, t_{4}\right)$, and group (SSC1, $\mathrm{SSC} 2, \mathrm{CON})$. Alterations in joint angles and ankle joint stiffness were analyzed with a repeated-measures ANOVA with the factors time (pre vs post), drop height ( $\mathrm{LH}, \mathrm{MH}$, $\mathrm{HH}$ ), group, and joint (hip, knee, ankle). In the case of significant $F$-values $(P<0.05)$, the effects were separately analyzed for each group using repeated-measures ANOVAs to identify interaction effects. The differences between values at selected points in time or between conditions were compared by Bonferroni corrected paired two-sided tests (Student's $t$-test). To identify the differences in adaptations between the two training groups ( $\mathrm{SSC} 1$ and $\mathrm{SSC} 2$ ), changes from pre- to post-measurement were expressed as percentage and Bonferroni-corrected unpaired two-sided tests were calculated. Correlations between changes in the $H / M$ ratio with changes in ankle joint stiffness were determined using the Pearson's correlation coefficient. SPSS 16.0 software was used for statistical analysis. All values are presented as group mean values \pm standard deviation in the text, while the results in the figures are given as mean values \pm standard error of mean (SEM) for display reasons.

\section{Results}

\section{Jump performance}

\section{Jump height and duration of ground contact}

In the pre-measurement, neither the jump height nor the duration of ground contact differed significantly in any condition ( $\mathrm{LH}, \mathrm{MH}, \mathrm{HH}$ ) between groups (in all cases $P>0.1$ ). The jump performance was influenced by the drop jump training in two ways: first, the duration of ground contact was significantly altered $\left(\right.$ time $_{\text {pre/post }}$ group $\left._{\text {consscissc: }}: F_{2,30}=5.1 ; P=0.011\right)$ and second, adaptations in jump height occurred (time pre/post group $\left._{\text {covsscissce }}: F_{2,30}=8.32 ; P=0.001\right)$. The control group did not change any of those parameters in the post-measurement. However, subjects of SSC1 and SSC2 showed clear but different adaptations; after
$\mathrm{SSC} 1$, there was an interaction of time and duration of ground contact $\left[F_{2,20}=4.3 ; P=0.028\right.$; Fig. 1(b)]. Although the time of ground contact was prolonged in each condition ( $\mathrm{LH}, \mathrm{MH}, \mathrm{HH})$, post hoc tests did not detect significant changes in any condition: LH $(+12 \pm 16 \mathrm{~ms}), \mathrm{MH}(+14 \pm 23 \mathrm{~ms})$, and $\mathrm{HH}$ condition $(+1 \pm 20 \mathrm{~ms}$; always $P>0.05)$. On the contrary, subjects in the SSC2 group reduced their duration of ground contact [time pre/post $; F_{1,10}=9.0 ; P=0.001 ;$ Fig. 1(b)]. Post hoc analysis revealed significantly decreased times of ground contact after SSC2 at LH $(P=0.002)$ and $\mathrm{HH}(P=0.028)$, but only a trend at $\mathrm{MH}$ $(P=0.06)$. The two training groups ( $\mathrm{SSC} 1$ and $\mathrm{SSC} 2)$ differed significantly with respect to their changes in duration of ground contact at LH $(P=0.004)$ and $\mathrm{MH}$ $(P=0.012)$ and showed a trend at HH $(P=0.06)$.

Regarding the jump height, performance improved in the SSC1 training group to a comparable extent in each condition [time pre/post; $F_{1,10}=14.6 ; P=0.003$; post hoc tests: $\mathrm{LH}, P=0.04 ; \mathrm{MH}, P=0.003 ; \mathrm{HH}$, $P=0.07$; Fig. 1(a)], but showed only a trend toward augmentation in the SSC2 group [time $F_{1,10}=4.27 ; P=0.066$; post hoc tests were not significant for any drop height; Fig. 1(a)]. When comparing the adaptations of the two training groups, significant differences could be detected at LH $(P=0.03)$ and $\mathrm{MH}(P=0.03)$.

\section{Performance index}

There was a significant interaction of time and group $\left[\right.$ time $_{\text {pre } / \text { post }} \times$ group $_{\mathrm{CON} / \mathrm{SSC} 1 / \mathrm{SSC} 2} ; F_{2,30}=6.7 ; P=0.004$; Fig. 1(c)]. The values in the control group remained unaltered in the post-measurement $\left(F_{1,10}=0.6\right.$; $P=0.44)$. In contrast, the performance indices were significantly enhanced in the two training groups (SSC1: $\quad F_{1,10}=9.5 ; \quad P=0.012 ; \quad$ SSC2: $\quad F_{1,10}=12.9$; $P=0.006)$. Thereby, the improvements expressed as percentage of the initial values were almost identical for the two groups ( $\mathrm{SSC} 1$ and $\mathrm{SSC} 2$ ) at all three drop heights $\quad(\mathrm{LH}: \quad \mathrm{SSC} 1=+10 \pm 17 \% \quad$ vs $\quad \mathrm{SSC} 2=+13$ $\pm 20 \% ; \quad P=0.79 ; \quad \mathrm{MH}: \quad \mathrm{SSC} 1=+13 \pm 17 \% \quad$ vS $\mathrm{SSC} 2=+14 \pm 13 \% ; P=0.92 ; \mathrm{HH}: \mathrm{SSC} 1=+19 \pm$ $23 \%$ vs $\mathrm{SSC} 2=+15 \pm 17 \% ; P=0.69$ ).

\section{$H_{\text {max }} / M_{\text {max }}$ ratios}

Pre- and post-measurements revealed no differences in the $H / M$ ratios (time pre/post $; F_{1,30}=1.2 ; P=0.29$ ) in any group (time pre/post $\times$ group $_{\mathrm{CON} / \mathrm{SSC} / \mathrm{SSC} 2}$; $\left.F_{2,30}=1.4 ; P=0.26\right)$ and at any stimulation point (time $_{\text {pre } / \text { post }} \times$ stimulation point $\mathrm{GND/SLR} ; F_{1,30}=0.5$; $P=0.49$ ). As reported in previous studies (Leukel et al., 2008a, 2008b), $H / M$ ratios were dependent on the drop height condition (drop height $\mathrm{LH}_{\mathrm{MH}, \mathrm{HH}}$; $\left.F_{2,60}=4.9 ; \quad P=0.011\right)$. This was due to a great modulation of the H-reflex amplitude when record- 

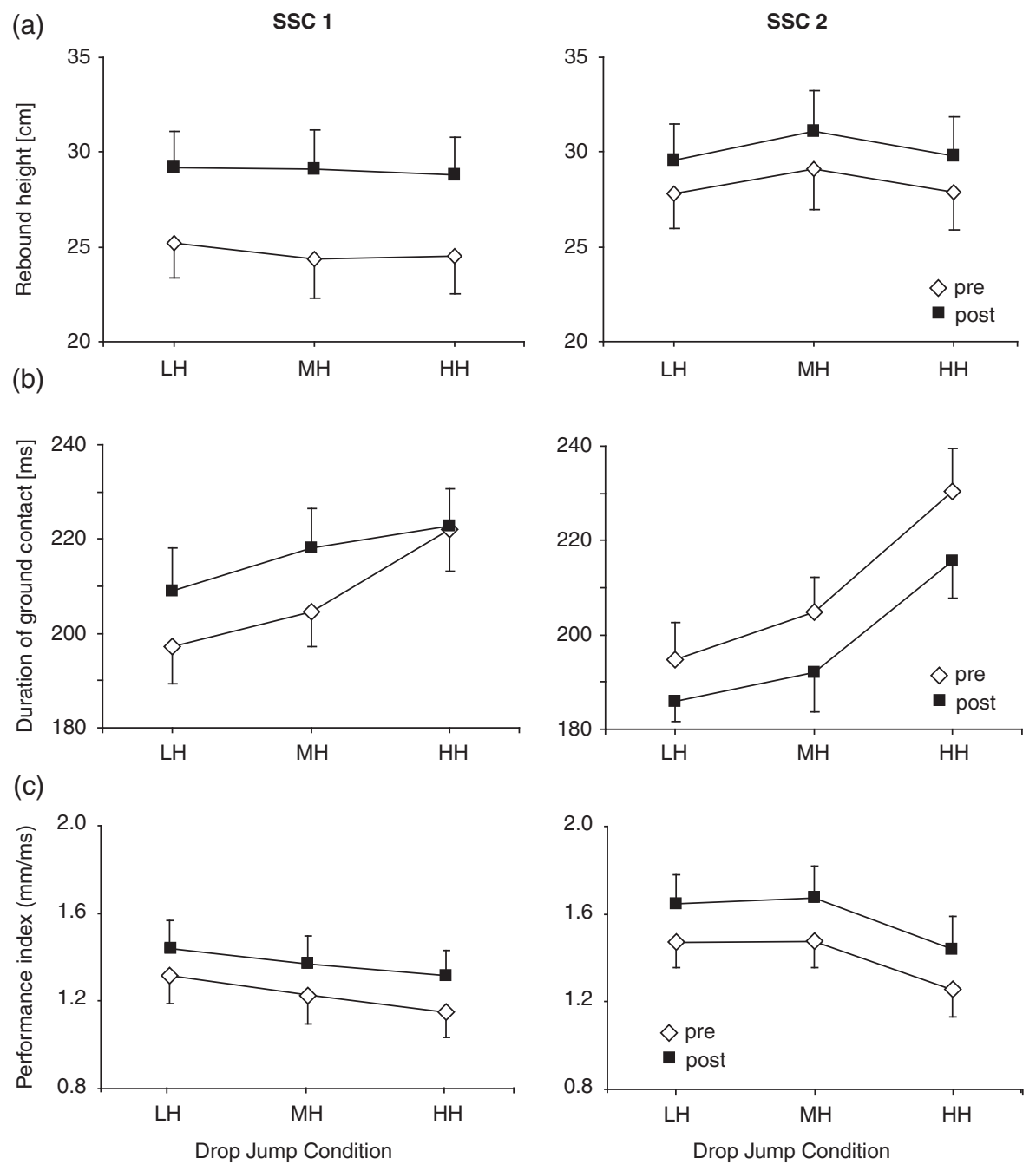

Fig. 1. Drop jump rebound height (a), duration of ground contact (b), and performance index (c) before $(\diamond)$ and after ( SSC1 and SSC2 training. (a) Subjects significantly increased their maximal rebound height from all three drop heights $(\mathrm{LH}=30 \mathrm{~cm} ; \mathrm{MH}=50 \mathrm{~cm} ; \mathrm{HH}=75 \mathrm{~cm})$ after SSC1. SSC2, on the other hand, caused only a trend toward augmented rebound heights. The duration of ground contact increased in the LH and MH but not the HH condition after SSC1 (b). In contrast, subjects reduced their duration of ground contact in all three jump conditions after SSC2. (c) The performance index was significantly enhanced after both SSC1 and SSC2. The improvements in performance index expressed as a percentage of the initial values were almost identical for the two training groups. SSC, stretch-shortening cycle; LH, low height; $\mathrm{MH}$, medium height; $\mathrm{HH}$, high height.

ings occurred at the time of the SLR (drop height $\left._{\mathrm{LH}, \mathrm{MH}, \mathrm{HH}} ; F_{2,60}=7.2 ; P=0.002\right) ; H / M$ ratios were greatest when jumping from $\mathrm{LH}$ and smallest when jumping from $\mathrm{HH}$ (Fig. 2). No task-specific modulation was observed when the H-reflex coincided with the duration of ground contact (drop height $\left._{\mathrm{LH}, \mathrm{MH}, \mathrm{HH}} ; F_{2,60}=0.7 ; P=0.50\right)$. No changes in any group (CON, SSC1 and SSC2), condition (LH, MH, HH and stance) or stimulation time (GND and SLR) were apparent in the maximal Mwaves from pre- to post-measurement (uncorrected Student's $t$-tests always $P>0.35$ ).

When the $H / M$ ratios measured during activity were expressed as a percentage of the $H / M$ ratios assessed at rest (standing) before and after SSC training, there were still no effects [method proposed in Voigt et al. (1998a); results not illustrated].

\section{Muscular activity}

Normalization to $M_{\max }$ of the muscular activity of the SOL muscle revealed a training-related change in activity, which was specific to the training group and the phase of the movement (time pre/post $\times$ phase $_{t 1-t 4} \times$ group $_{\mathrm{CON} / \mathrm{SSCl} / \mathrm{SSC} 2} ; F_{6,180}=4.1 ; P=0.001$; Fig. 3). Following SSC1 training, muscular activity of the SOL did not change in the early phase of the drop jump (pre-activation and early reflex phase: -100 to $+70 \mathrm{~ms} ; 0 \mathrm{~ms}$ corresponds to the instant of ground contact). In contrast, muscular activity during 

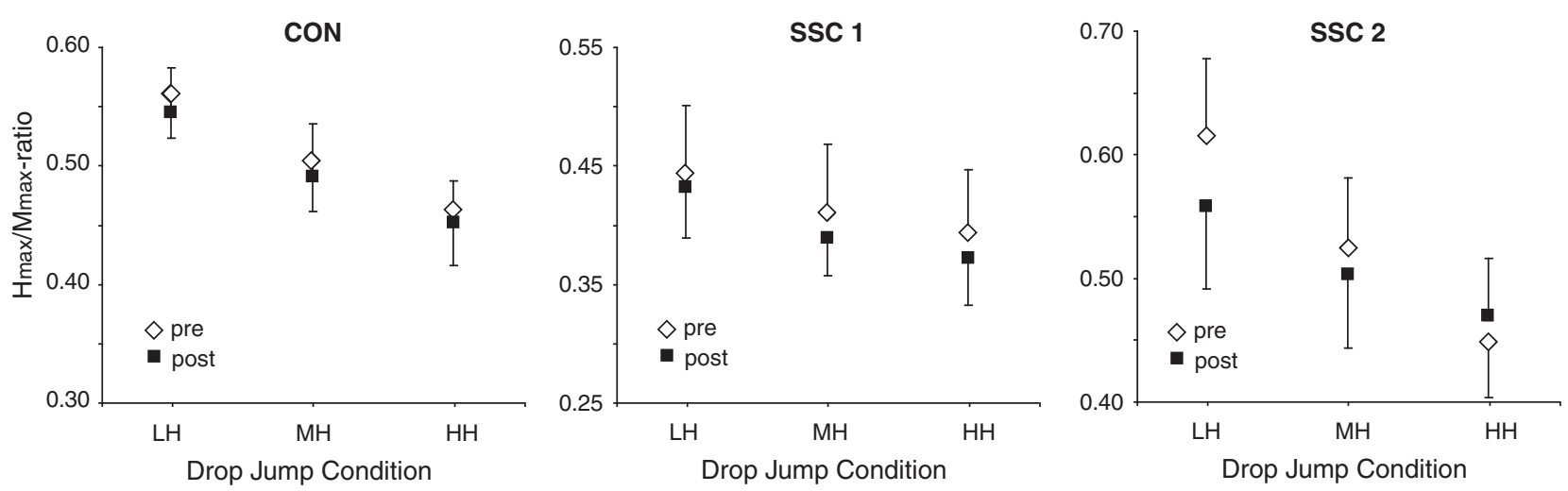

Fig. 2. $H_{\max } / M_{\max }$ ratios of the control (CON), the SSC1 and SSC2 group at jumps from different drop heights [low height $(30 \mathrm{~cm})=\mathrm{LH}$; moderate height $(50 \mathrm{~cm})=\mathrm{MH}$; and high height $(75 \mathrm{~cm})=\mathrm{HH}]$. Before training $(\diamond)$, all three groups demonstrated a significant drop-height-specific H-reflex modulation. In the post-measurement ( $\boldsymbol{\square})$, the same results were obtained except for the SSC2 group, which did not show a significant drop-height-dependent H-reflex modulation any more. SSC, stretch-shortening cycle; $\mathrm{LH}$, low height; $\mathrm{MH}$, medium height; $\mathrm{HH}$, high height.

the late phase of ground contact $(70-170 \mathrm{~ms})$ increased (time $_{\text {pre } / \text { post }} \times$ phase $_{t 1-t 4} ; F_{3,30}=5.0 ; P=0.007 ;$ Fig. 3). Following SSC2 training, the muscular activity did not change during the late phase of ground contact but in the early reflex phase $\left(\right.$ time $_{\text {pre/post }} \times$ phase $_{t 1-t 4}$; $F_{3,30}=5.1 ; P=0.006$; Fig. 3).

Figure 4 illustrates one participant's data before and after SSC1 training and pre- and postmeasurement data of one subject from the SSC2 group. It can clearly be seen that muscular adaptations occurred at different times with regard to the training intervention; after SSC1, muscular activity increased toward push-off while SSC2 resulted in an increased muscular activity at the time of the SLR. These neuromuscular adaptations probably influenced the pattern of the ground reaction forces; after $\mathrm{SSC1}$, a prolonged time of ground contact was apparent whereas the opposite was true for $\mathrm{SSC} 2$ (Fig. 4).

In the SSC1 group, the RF muscle behaved similar to the SOL, showing unchanged activity in the early phase of drop jump and increased neural drive in the later part of ground contact $\left(\right.$ time $_{\text {pre/pos }} \times$ phase $\left._{t 1-t 4} ; \quad F_{3,30}=4.7 ; \quad P=0.008\right)$. In the SSC2 group, the activity of the RF was not altered after training. No training-induced changes at all were seen in the GM and the TA as well as in all muscles tested in the control group (not illustrated; all statistical values above $P=0.23$ ).

\section{Motion analysis}

Unfortunately, the signals of the goniometers used in the SSC 2 could not be analyzed. Although the signals looked well online, later analysis revealed measurement errors, which were caused by a loose contact within the cable connecting the goniometer to the $\mathrm{A} /$ $\mathrm{D}$ converter. Thus, the kinematic data are missing for the SSC2 group.
Even though the kinematic data are solely available for the control and SSC1 group, it is nonetheless illustrated as it may provide important additional information due to the following two reasons: first, the longitudinal data of the $\mathrm{SSC} 1$ group can be better compared with other SSC-training studies focusing on changes in lower leg stiffness rather than the neuromuscular activity (Hunter \& Marshall, 2002; Kubo et al., 2007). Second, the cross-sectional data obtained in the pre-measurement highlight the previously proposed relationship between H-reflex excitability and lower leg stiffness (Dietz et al., 1979; Dyhre-Poulsen et al., 1991; Gollhofer et al., 1992; Voigt et al., 1998b; Komi, 2003; Hobara et al., 2007).

\section{Kinematics}

SSC1 training significantly increased maximal joint flexion during the eccentric phase of the drop jump (countermovement), whereas there were no changes in the CON group (time $\times$ group effect; $F_{1,18}=8.77$, $P=0.008)$. In the training group, alterations in the depth of countermovement were most pronounced for the ankle and knee joint and only minor for the hip (time $_{\text {pre } / \text { post }} \times$ joint; $F_{2-12}=8.9 ; P<0.004$; Fig. 5). Most interestingly, training-induced changes occurred solely in jumps from the lower drop heights, i.e., from 30 and $50 \mathrm{~cm}$, but not from $75 \mathrm{~cm}$ (condition: $F_{2-12}=37.4 ; P<0.001$; Fig. 5).

\section{Joint stiffness}

Ankle joint stiffness did not change after SSC1 training (time $\times$ group effect; $F_{1,18}=0.00 ; P=0.99$ ). However, in pre- and post-measurement, drop height was shown to have a major impact on ankle joint stiffness resulting in reduced stiffness when jumping from greater heights (drop height ${ }_{\mathrm{LH}, \mathrm{MH}, \mathrm{HH}} ; F_{2,34}=20.78$; $P<0.001)$. 
SSC 1

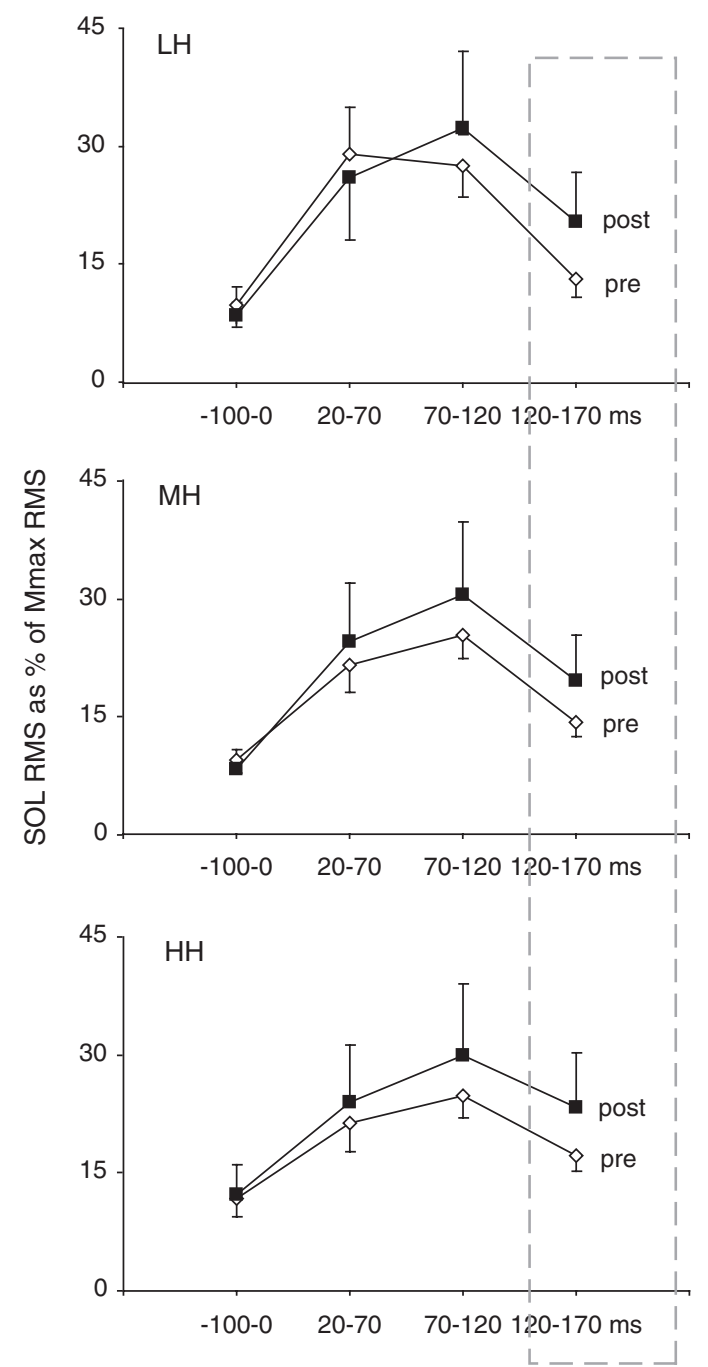

SSC 2

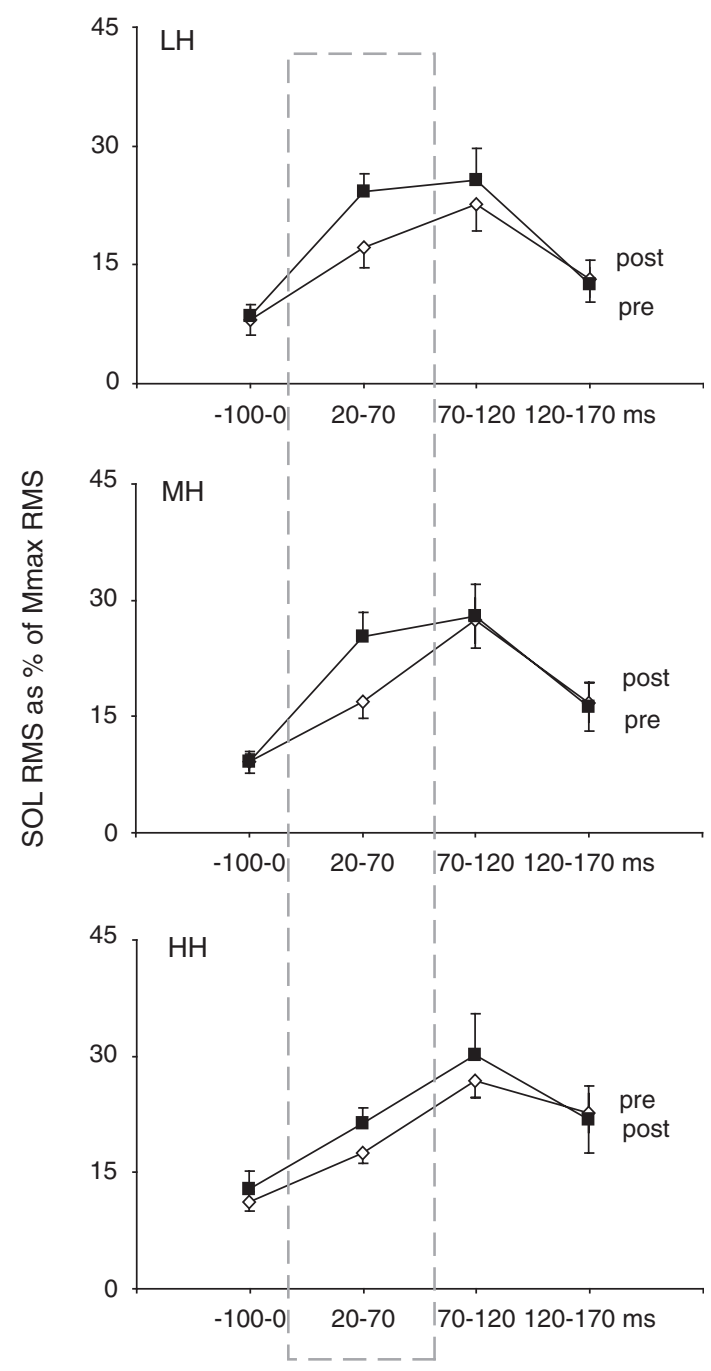

Fig. 3. Muscular activity of the M. soleus during drop jumps from $30 \mathrm{~cm}(\mathrm{LH}), 50 \mathrm{~cm}(\mathrm{MH})$ and $75 \mathrm{~cm}(\mathrm{HH})$ in premeasurement $(\diamond)$ and post-measurement $(\boldsymbol{\square})$ of SSC1- and SSC2-trained subjects. After SSC1, muscular activity remained unchanged in the pre-activation phase ( -100 to $0 \mathrm{~ms}$ before touch down) and the early duration of ground contact (20$70 \mathrm{~ms}=$ "early reflex phase"). In later phases of ground contact (70-120 and 120-170 ms) muscular activity increased following SSC1 training. In contrast, changes after SSC2 were most pronounced shortly after ground contact in the "early reflex phase" but displayed no significant changes in any other phase. SSC, stretch-shortening cycle; LH, low height; MH, medium height; $\mathrm{HH}$, high height.

Correlation between ankle joint stiffness and $H / M$ ratios from different drop heights

Data assessed in the pre-measurement revealed that the changes in ankle joint stiffness due to increases in drop height (from $\mathrm{LH}$ to $\mathrm{MH}$ and from $\mathrm{LH}$ to $\mathrm{HH}$ ) were positively correlated with changes in the $H / M$ ratio (Fig. 6). This means that subjects who reduced their ankle joint stiffness largely when jumping from MH instead of LH concomitantly demonstrated great reductions in their $H / M$ ratios $[r=0.66$; $P=0.003$; Fig. 6(a)]. Similar results were obtained when changes in ankle stiffness and $H / M$ ratios were correlated when switching from $\mathrm{LH}$ to $\mathrm{HH}[r=0.56$;
$P=0.015$; Fig. 6(b)]. The post-measurement data partially confirmed this observation resulting in a significant correlation between changes in ankle joint stiffness and changes of $H / M$ ratios when switching from $\mathrm{LH}$ to $\mathrm{MH}(r=0.50 ; P=0.036)$ but not from $\mathrm{MH}$ to $\mathrm{HH}(r=0.22 ; P=0.37$; post-measurement data are not displayed).

\section{Discussion}

It was hypothesized that the changes in neuromuscular activity and jumping technique in response to SCC training are specifically linked to the applied 
(a) SSC1

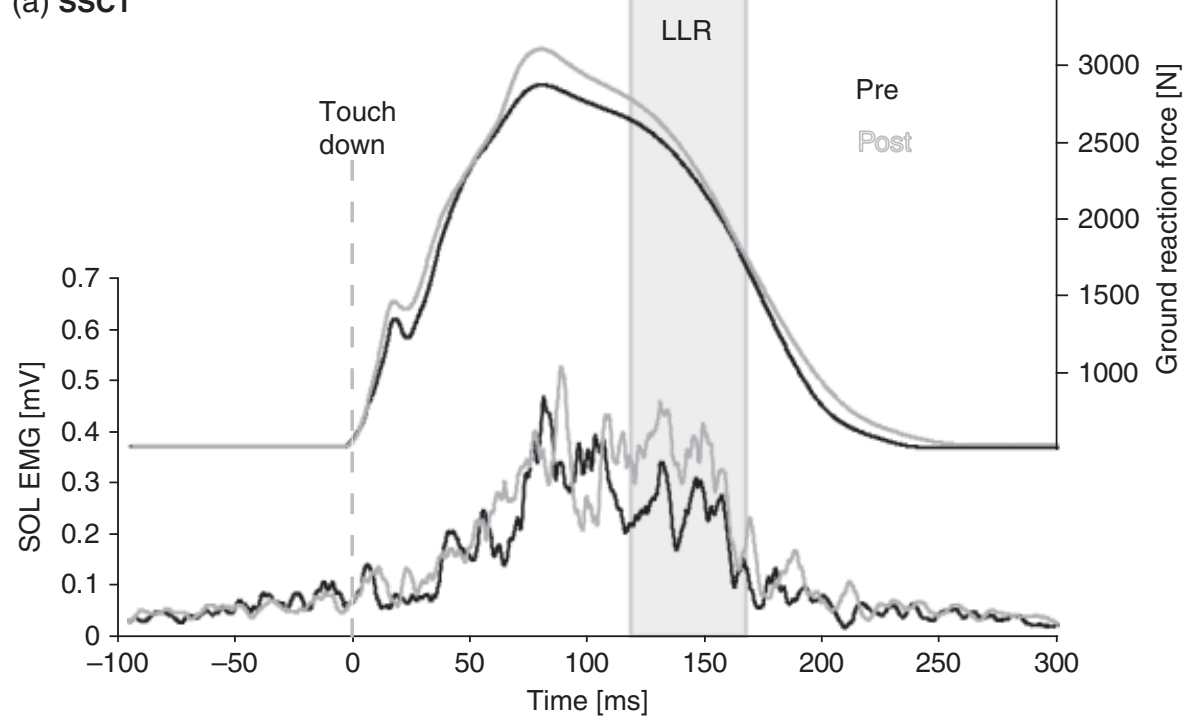

(b) Ssc2

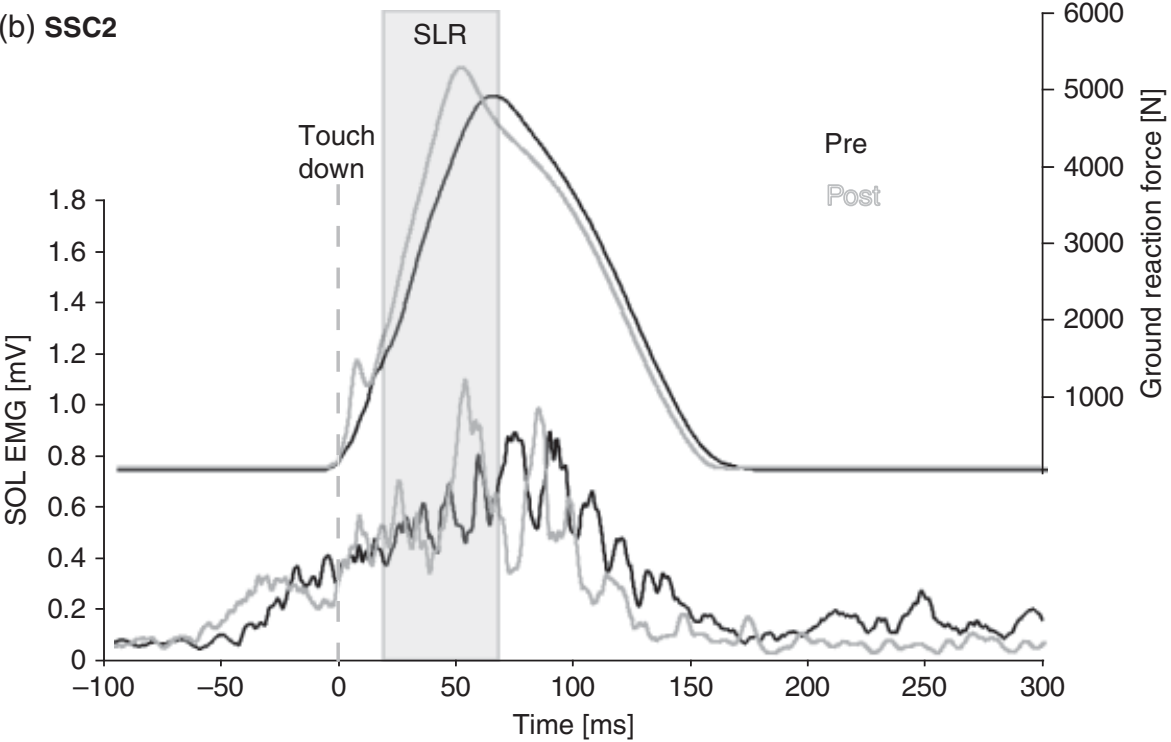

Fig. 4. Muscular activity of the M. soleus during drop jumps from $30 \mathrm{~cm}$ of one SSC1-trained subject (a) and one SSC2-trained subject (b). After SSC1, muscular activity remained unchanged in the pre-activation phase but was enhanced in later phases of ground contact (120-170 ms; gray-shaded box). At the same time, the duration of ground contact was prolonged (a). In contrast, electromyogram changes after SSC2 were most pronounced shortly after ground contact in the "early reflex phase" (20-70 ms; gray-shaded box) but displayed no significant changes in any other phase (b). The duration of ground contact was reduced after SSC2. LLR, long latency response; SLR, short latency response; SSC, stretch-shortening cycle.

drop heights. In particular, changes in the muscular activity and H-reflex excitability at the time of the SLR together with alterations in performance-related biomechanical parameters of the drop jumps were expected to be determined by the applied stretch loads during the training interventions. The results of the present study confirm our initial hypotheses.

\section{Training-specific adaptations after SSC1}

After SSC1 training, there were neither changes in the muscular activity of SOL and GM nor in the antagonist TA or the H-reflex at the time of the SLR.
Therefore, the enhanced rebound heights following SSC1 training cannot be explained by alterations of the early stretch reflex component in all likelihood. Also, the increased jump height cannot be attributed to an enhanced ankle joint stiffness in the eccentric phase, as this parameter remained unchanged after $\mathrm{SSC} 1$ training. This result seems reasonable as the spinal H-reflex activity and the ankle joint stiffness were significantly correlated for the jumps from different drop heights (Fig. 6). As reported previously (Leukel et al., 2008a, 2008b) and confirmed in the present study, the H-reflex excitability was decreased with increasing drop heights. At the same 

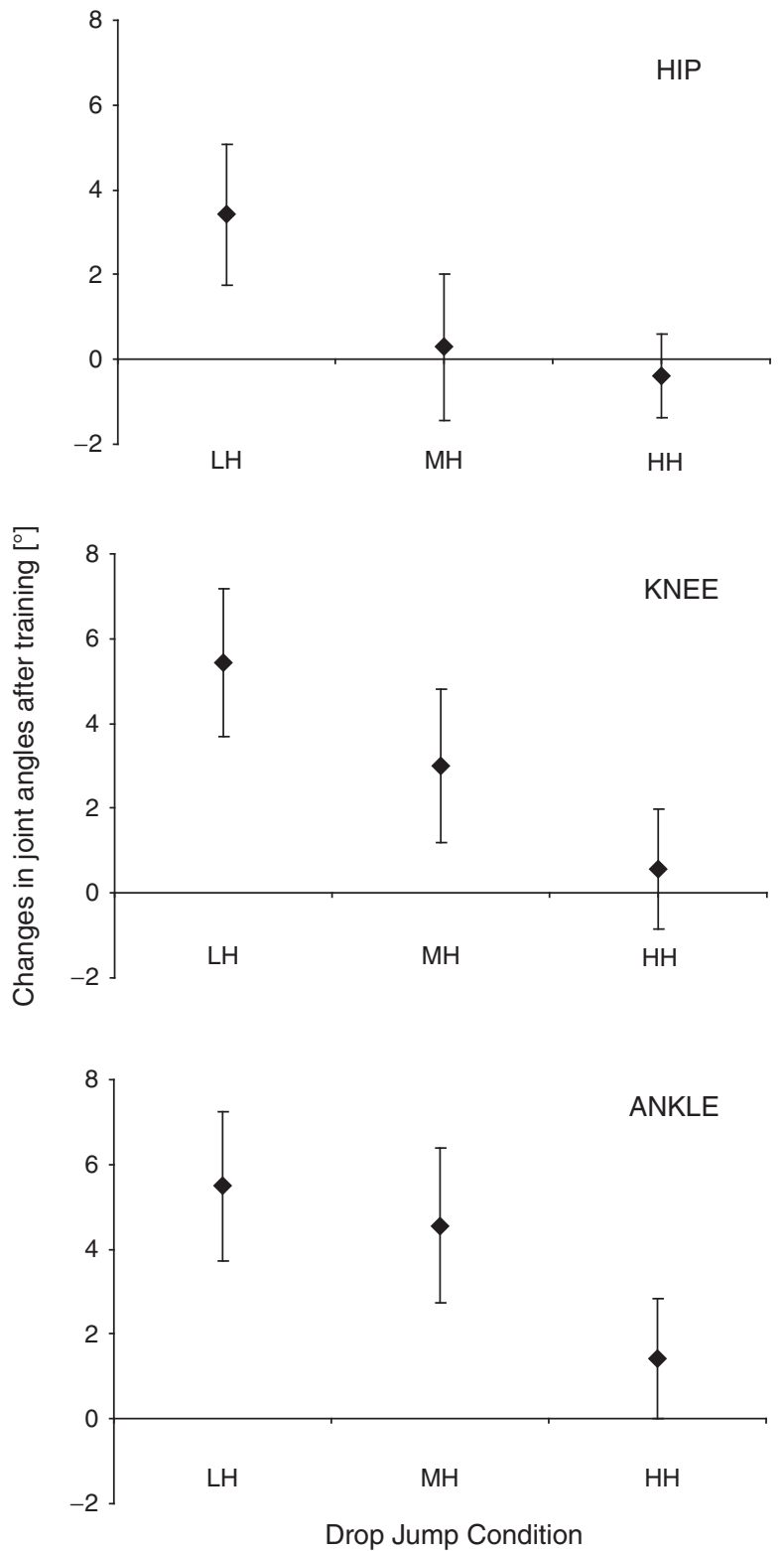

Fig. 5. Changes in joint angles (hip, knee, and ankle angles) during drop jumps from $30 \mathrm{~cm}(\mathrm{LH}), 50 \mathrm{~cm}(\mathrm{MH})$, and $75 \mathrm{~cm}$ $(\mathrm{HH})$ after SSC1 training. Subjects demonstrated greater hip, knee, and ankle flexion after training when tested in the LH condition. Knee and ankle angles also decreased in the $\mathrm{MH}$ condition. No changes occurred in the $\mathrm{HH}$ condition. Positive values indicate greater joint flexion. SSC, stretchshortening cycle; $\mathrm{LH}$, low height; $\mathrm{MH}$, medium height; $\mathrm{HH}$, high height.

time, the ankle joint stiffness was reduced. This correlation may strengthen the long-held assumption (Dietz et al., 1979; Dyhre-Poulsen et al., 1991; Gollhofer et al., 1992; Voigt et al., 1998b; Komi, 2003; Hobara et al., 2007) that spinal reflexes at the time of the SLR affect the ankle joint stiffness during SSC movements.

Although SSC1-trained subjects did not alter their ankle joint stiffness, they significantly increased their countermovement, i.e., they made use of greater ankle and knee flexion in the eccentric phase of the drop jump. As subjects displayed an unaltered EMG activity in agonists and antagonists in this early period after ground contact but a greater joint flexion, SSC1 training may have changed the EMG to torque relationship. It might be speculated that the extensive eccentric loads in jumps from $\mathrm{HH}$ could have led to adaptations within the muscle and/or tendon. For instance, evidence from animal studies suggests that eccentric resistance training increases muscle fascicle length by increasing the number of sarcomeres in series (Morgan \& Allen, 1999; Butterfield \& Herzog, 2006). Exercise-induced changes in muscle fascicle length were also demonstrated in humans (Reeves et al., 2005, 2009; Blazevich et al., 2007) and could therefore explain the altered torqueangle relationship in the present study. With the present methodology, this issue cannot be answered but it has to be underlined that the present training was restricted to 4 weeks in order to induce predominantly neural rather than tendo-muscular adaptations (Moritani \& deVries, 1979).

Apart from the greater countermovement, subjects also demonstrated significant increases in the ground contact time when jumping from $\mathrm{LH}$ and $\mathrm{MH}$ after training. However, most relevant with respect to improved jump performance may be the observation of increased muscular activity in the soleus and RF muscles in the late phase of the drop jump. This increased muscular activity shortly before take-off combined with the longer distance (greater depth of countermovement) and time for acceleration (ground contact time) probably enabled subjects to increase their take-off velocity.

\section{Adaptations after SSC2 training}

The SSC2 training resembled the SSC1 training but incorporated only $\mathrm{LH}$ drop heights $(30 \mathrm{~cm})$. We hypothesized that the SSC2 training should exert a stronger influence on the neuromuscular activity at the time of the SLR because subjects would not have to apply similar strong inhibitory mechanisms in the eccentric phase than during jumps from high drop heights. The normalized activity of the SOL muscle was indeed enhanced at the time of the SLR. However, the $H / M$ ratios were not augmented. Thus, it might be argued that a more efficient (e.g. removal of inhibition) Ia afferent transmission to the $\alpha$-motoneurons was not responsible for the increased muscular activity. Alternatively, the increase of the normalized soleus background EMG might be caused by enhanced supraspinal contributions. Previously, we demonstrated that during hopping the muscular activity at the time of the SLR ("early reflex phase") is not only exclusively generated by stretch reflex responses but also depends on the 

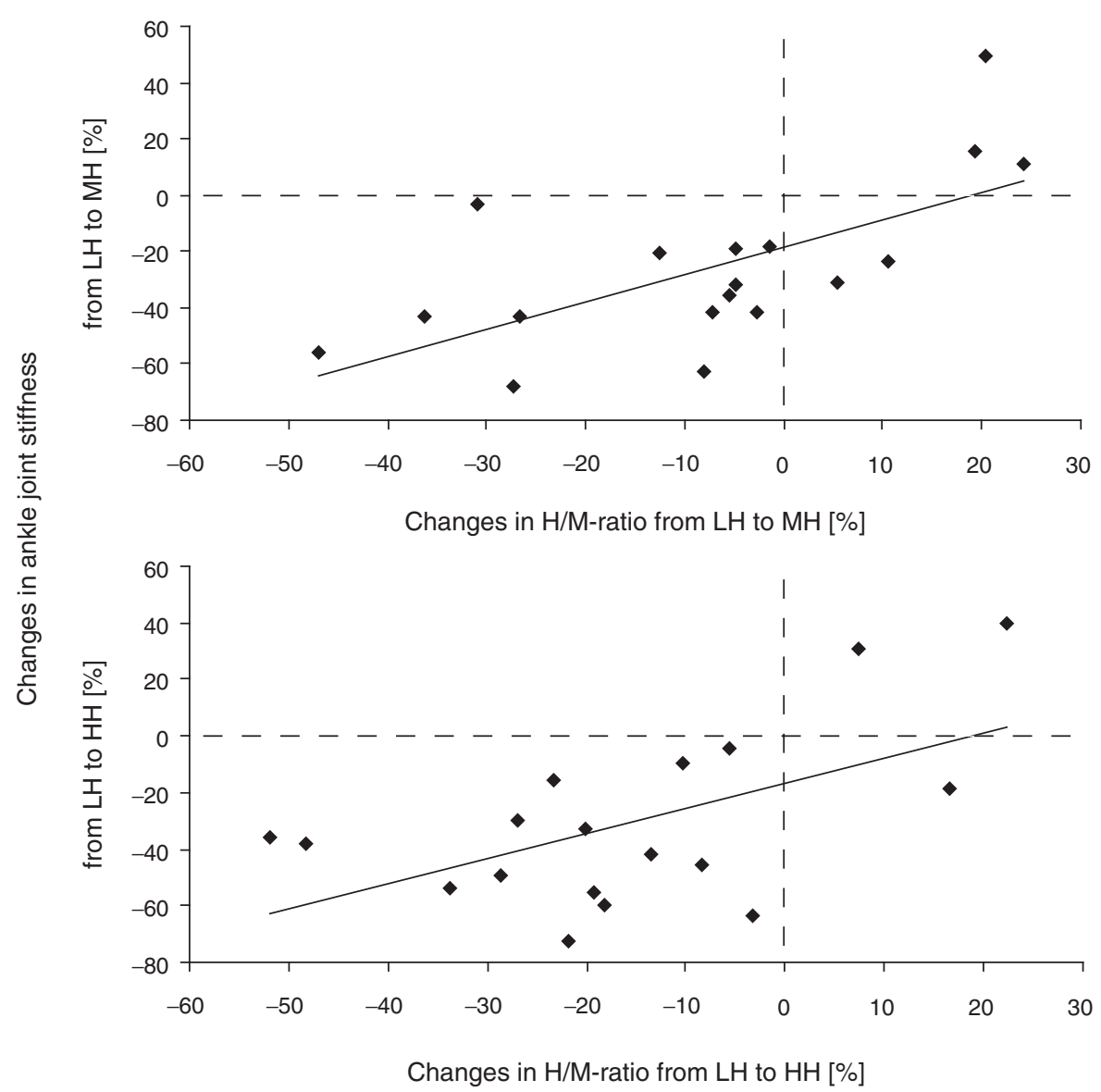

Fig. 6. Correlation of drop-height-dependent changes in the ankle joint stiffness and changes in the $H / M$ ratios. (a) Subjects of SSC1 and CON demonstrated a correlation when comparing ankle joint stiffness and $H / M$ ratios in the LH and MH conditions $(r=0.66 ; P=0.003)$ as well as when comparing ankle joint stiffness and $H / M$ ratios in the LH and HH conditions $(r=0.56 ; P=0.015)$ (b). SSC, stretch-shortening cycle; LH, low height; $\mathrm{MH}$, medium height; HH, high height.

contribution of cortical centers (Zuur et al., 2010). With the methods used in the present study, the site and the mechanism(s) of adaptation leading to an enhanced neural drive cannot be identified. However, irrespective of the exact mechanism(s), it is very likely that this augmented neural drive at the SLR enabled the subjects to reduce their times of ground contact and to augment their performance indices. This strategy, however, does not seem beneficial in increasing the rebound height, as there was only a trend toward augmentation.

\section{"Movement instruction" vs "constraints of the motor task"}

In all training and testing sessions, the subjects were instructed to "rebound as fast and as high as possible," i.e., to reduce the duration of ground contact and to enhance the jump height. However, subjects of the $\mathrm{SSC} 1$ group enhanced their rebound height but displayed prolonged times of ground contact. In contrast, SSC2-trained subjects reduced the duration of ground contact but showed non-significant changes in the rebound height. Apparently, no group put the movement instructions completely into action. Previously, Hunter and Marshall (2002) presumed that if subjects were not advised by instructions like: "Keep the duration of ground contact as short as possible," or "Bend your knees very little," subjects would automatically adapt their jumping strategy in the direction of a lower leg stiffness, greater depth of countermovement, and longer ground contact times. This assumption resulted from the observation that non-instructed subjects adopted such a strategy after 10 weeks of drop jump training from different drop heights $(30,45,60$, 75 , and $90 \mathrm{~cm}$ ). However, other studies could not confirm this hypothesis. For instance, Kubo et al. (2007) did not instruct their subjects to rebound as fast as possible but observed enhanced lower leg stiffness without changes in the angular velocities. On the first view, the outcome after drop jump training seems therefore arbitrary. However, when considering the drop heights applied during training, it becomes obvious that the two interventions [SSC1 and Hunter \& Marshall (2002)] incorporating high drop heights (above $75 \mathrm{~cm}$ ) led to greater depth of the 
countermovement, longer duration of ground contact and unchanged or decreased lower leg stiffness. In contrast, training from lower drop heights (under $60 \mathrm{~cm}$ ) resulted in enhanced lower leg stiffness (Kubo et al., 2007), decreased countermovement, and shorter duration of ground contact [SSC2 and $\mathrm{Bu}-$ beck et al. (2003); de Villarreal et al. (2008)]. These observations show the importance of the drop height applied during the training. Consequently, it can be concluded that, at least in some cases, the direction of adaptation is not guided mainly by the instruction but by the task itself. This was most evident in the $\mathrm{SSC} 1$ group in which the jumping technique in the $\mathrm{LH}$ and $\mathrm{MH}$ conditions was altered in direction of the $\mathrm{HH}$ condition, whereas alterations in the $\mathrm{HH}$ condition were minor or non-existent. Therefore, we can speculate that the jumping strategy used in the $\mathrm{HH}$ condition was transferred to the lower height conditions. The reason for the strong influence of the $\mathrm{HH}$ condition on the jump technique may lie in the responsibility of the neuromuscular system to avoid overload injuries. Kjaer (2004) reported the maximal loading for several tendons and demonstrated that the Achilles and patella tendons work in ranges relatively close to their point of failure. Especially when jumping, very high forces are exerted on the tendons (Fukashiro et al., 1995; Kjaer et al., 2005). Thus, neural control may adapt to high drop heights in terms of a reduced spinal excitability to reduce the stiffness of the muscle-tendon complex and avoid the occurrence of overload injuries (Kyrolainen \& Komi, 1995; Komi \& Gollhofer, 1997; Komi, 2003; Leukel et al., 2008b). Interestingly, elderly people apply the "high drop height technique" already in jumps from low heights. Compared with young subjects, they showed less muscular activity during the pre-activation and early breaking phase (eccentric phase = "early reflex phase") of drop jumps but increased activity in the push-off phase (Hoffren et al., 2007). This technique resulted in a lower stress of the tendo-muscular system in the eccentric phase. In young subjects, training incorporating high drop heights (SSC1) may transfer such a "prevention strategy" to other jump conditions ( $\mathrm{LH}, \mathrm{MH})$ despite the instruction to rebound as fast as possible. The discovery of a taskdependent "overruling" of the movement instruction is important for subsequent plyometric training interventions. It is not only sufficient to ask the subjects to perform a certain technique (e.g. realization of short ground contact times) but also to provide the optimal loading (drop height).

\section{Functional considerations}

The present longitudinal study demonstrated a clear relationship between the stretch load (drop height) and the resulting adaptations in neuromuscular ac- tivity and performance. Previous cross-sectional studies proposed that in order to maximize the mechanical output of knee extensors and plantar flexors during drop jump training, athletes should utilize a "bounce drop jump technique," i.e., to reverse the downward velocity into an upward one as soon as possible after landing (Bobbert et al., 1987a). This "recommendation" was founded on the essential need for effective storage of elastic energy in the transition from the eccentric to the concentric phase (Kyrolainen \& Komi, 1995; Komi, 2003). Regarding the drop height, it was suggested to train from low drop heights $(20-40 \mathrm{~cm})$ to avoid a sharp heel impact in the eccentric phase (Bobbert et al., 1987b; Lees \& Fahmi, 1994). The SSC2 training in the present study resembled such a training and caused enhanced neuromuscular activity at the time of the SLR, which probably enabled subjects to reduce their times of ground contact but proved ineffective in increasing the rebound height. Conversely, SSC1-trained subjects enhanced neuromuscular activity toward the push-off, which not only led to improved vertical rebound heights but also prolonged times of ground contact. In line with Hunter and Marshall (2002), the incorporation of high drop heights led to a greater depth of the countermovement. Thus, SSC1 and SSC2 caused distinct neuromuscular and biomechanical adaptations, which were dependent on the training regime, or more specifically, the drop height (or stretch load). To compare the efficiency of the two training regimes, the training-related changes in performance were analyzed. For this purpose, the workload was timenormalized, i.e., the rebound height was divided by the duration of ground contact and expressed as the performance index (performance index $=$ rebound height/contact time $\times$ s/m; Bruhn et al., 2004; Taube et al., 2007). This analysis revealed that both SSC interventions led to significant and - most interestingly - comparable improvements. As the gain in performance was not different after SSC1 and SSC2, it might be speculated that the training outcomes are limited by the amount of reactive performance the tendo-muscular system can execute. The decision whether to apply SSC1 or SSC2 should therefore strongly depend on the specific demands of the particular sports discipline. High drop heights should be implemented into the training regime if the goal is to maximize the rebound height and if the duration of ground contact is not a limiting factor of performance. On the other hand, low drop heights should be preferred to maximize power output in time critical disciplines. This drop-height-dependent specificity of the neuromuscular adaptations may therefore also partly explain the great variations in training efficiency of SSC interventions reported in a recent meta-analytical review (Markovic, 2007). 


\section{Perspectives}

The present study is the first one to highlight the fact that differences in the drop height applied during SSC training determines the neuromuscular adaptations. As the neuronal control in turn seems to strongly affect the performancerelated parameters, this finding may be of great relevance not only to understand the task specificity of neural adaptations in SSC movements but may also help to conceptualize appropriate training regimes.

Key words: H-reflex, drop jump, peripheral nerve stimulation, plyometric training.

\section{Acknowledgement}

This project was funded by the Bundesinstitut für Sportwissenschaft (BISp; Germany) and by the Bundesamt für Sport (BASPO; Switzerland).

\section{References}

Aagaard P, Simonsen EB, Andersen JL, Magnusson P, Dyhre-Poulsen P.

Neural adaptation to resistance training: changes in evoked V-wave and H-reflex responses. J Appl Physiol 2002: 92: 2309-2318.

Blazevich AJ, Cannavan D, Coleman $\mathrm{DR}$, Horne S. Influence of concentric and eccentric resistance training on architectural adaptation in human quadriceps muscles. J Appl Physiol 2007: 103: 1565-1575.

Bobbert MF. Drop jumping as a training method for jumping ability. Sports Med 1990: 9: 7-22.

Bobbert MF, Huijing PA, Ingen Schenau GJ. Drop jumping. I. The influence of jumping technique on the biomechanics of jumping. Med Sci Sports Exerc 1987a: 19: 332-338.

Bobbert MF, Huijing PA, Ingen Schenau GJ. Drop jumping. II. The influence of dropping height on the biomechanics of drop jumping. Med Sci Sports Exerc 1987b: 19: 339-346.

Bruhn S, Kullmann N, Gollhofer A. The effects of a sensorimotor training and a strength training on postural stabilisation, maximum isometric contraction and jump performance. Int J Sports Med 2004: 25: 56-60.

Bubeck D, Sialis I, Gollhofer A. Influence of different training amounts on the mechanical and neuromuscular parameters of the stretch shortening cycle. In: Müller E, Schwameder H, Zallinger G, Fastenbauer V. eds. Abstract Book of the 8th Annual Congress of the European College of Sport Science. Salzburg, 2003: p. 392.

Butterfield TA, Herzog W. The magnitude of muscle strain does not influence serial sarcomere number adaptations following eccentric exercise. Pflugers Arch 2006: 451: 688700.

Cardinale M., Bosco C. The use of vibration as an exercise intervention. Exerc Sport Sci Rev 2003: 31: 3-7. de Villarreal ES, Gonzalez-Badillo JJ, Izquierdo M. Low and moderate plyometric training frequency produces greater jumping and sprinting gains compared with high frequency. J Strength Cond Res 2008: 22: 715-725.

Dietz V, Schmidtbleicher D, Noth J. Neuronal mechanisms of human locomotion. J Neurophysiol 1979: 42: 1212-1222.

Dyhre-Poulsen P, Simonsen EB, Voigt M. Dynamic control of muscle stiffness and $\mathrm{H}$ reflex modulation during hopping and jumping in man. $\mathbf{J}$ Physiol 1991: 437: 287-304.

Fukashiro S, Komi PV, Jarvinen M, Miyashita M. In vivo Achilles tendon loading during jumping in humans. Eur J Appl Physiol Occup Physiol 1995: 71: 453-458.

Gollhofer A, Strojnik V, Rapp W, Schweizer L. Behaviour of triceps surae muscle-tendon complex in different jump conditions. Eur J Appl Physiol Occup Physiol 1992: 64: 283-291.

Hobara H, Kanosue K, Suzuki S. Changes in muscle activity with increase in leg stiffness during hopping. Neurosci Lett 2007: 418: 55-59.

Hoffer JA, Andreassen S. Regulation of soleus muscle stiffness in premammillary cats: intrinsic and reflex components. J Neurophysiol 1981: 45: 267-285.

Hoffren M, Ishikawa M, Komi PV. Agerelated neuromuscular function during drop jumps. J Appl Physiol 2007: 103: 1276-1283.

Hunter JP, Marshall RN. Effects of power and flexibility training on vertical jump technique. Med Sci Sports Exerc 2002: 34: 478-486.

Kawakami Y, Muraoka T, Ito S, Kanehisa $\mathrm{H}$, Fukunaga $\mathrm{T}$. In vivo muscle fibre behaviour during countermovement exercise in humans reveals a significant role for tendon elasticity. J Physiol 2002: 540: 635-646.

Kjaer M. Role of extracellular matrix in adaptation of tendon and skeletal muscle to mechanical loading. Physiol Rev 2004: 84: 649-698.
Kjaer M, Langberg H, Miller BF, Boushel R, Crameri R, Koskinen S, Heinemeier K, Olesen JL, Dossing S, Hansen M, Pedersen SG, Rennie MJ, Magnusson P. Metabolic activity and collagen turnover in human tendon in response to physical activity. J Musculoskelet Neuronal Interact 2005: 5: 41-52.

Komi PV. Stretch-shortening cycle. In: Komi PV., ed. Strength and power in sport. Oxford: Blackwell Science, 2003: 184-202.

Komi PV, Gollhofer A Stretch reflex can have an important role in force enhancement during SSC-exercise. J Appl Biomech 1997: 13: 451-460.

Kubo K, Morimoto M, Komuro T, Yata H, Tsunoda N, Kanehisa H, Fukunaga T. Effects of plyometric and weight training on muscle-tendon complex and jump performance. Med Sci Sports Exerc 2007: 39: 1801-1810.

Kuitunen S, Avela J, Kyrolainen H, Nicol $\mathrm{C}, \mathrm{Komi}$ PV. Acute and prolonged reduction in joint stiffness in humans after exhausting stretch-shortening cycle exercise. Eur J Appl Physiol 2002: 88: 107-116.

Kyrolainen H, Komi PV. Differences in mechanical efficiency between powerand endurance-trained athletes while jumping. Eur J Appl Physiol Occup Physiol 1995: 70: 36-44.

Lees A, Fahmi E. Optimal drop heights for plyometric training. Ergonomics 1994: 37: 141-148.

Leukel C, Gollhofer A, Keller M, Taube W. Phase- and task-specific modulation of soleus H-reflexes during drop-jumps and landings. Exp Brain Res 2008a: 190: 71-79.

Leukel C, Taube W, Gruber M, Hodapp $\mathrm{M}$, Gollhofer A. Influence of falling height on the excitability of the soleus H-reflex during drop-jumps. Acta Physiol (Oxf) 2008b: 192: 569-576.

Malatesta D, Cattaneo F, Dugnani S, Maffiuletti NA. Effects of electromyostimulation training and volleyball practice on jumping ability. J Strength Cond Res 2003: 17: 573-579. 
Markovic G. Does plyometric training improve vertical jump height? A metaanalytical review. Br J Sports Med 2007: 41: 349-355.

Morgan DL, Allen DG. Early events in stretch-induced muscle damage. J Appl Physiol 1999: 87: 2007-2015.

Moritani T, de Vries HA. Neural factors versus hypertrophy in the time course of muscle strength gain. Am J Phys Med 1979: 58: 115-130.

Reeves ND, Maganaris CN, Longo S, Narici MV. Differential adaptations to eccentric versus conventional resistance training in older humans. Exp Physiol 2009: 94: 825-833.

Reeves ND, Maganaris CN, Narici MV. Plasticity of dynamic muscle performance with strength training in elderly humans. Muscle Nerve 2005: 31: 355-364.
Taube W, Kullmann N, Leukel C, Kurz O, Amtage F, Gollhofer A. Differential reflex adaptations following sensorimotor and strength training in young elite athletes. Int J Sports Med 2007: 28: 999-1005.

Taube W, Leukel C, Schubert M, Gruber M, Rantalainen T, Gollhofer A. Differential modulation of spinal and corticospinal excitability during drop jumps. J Neurophysiol 2008: 99: 12431252.

Voigt M, Chelli F, Frigo C. Changes in the excitability of soleus muscle short latency stretch reflexes during human hopping after 4 weeks of hopping training. Eur J Appl Physiol Occup Physiol 1998a: 78: 522-532.

Voigt M, Dyhre-Poulsen P, Simonsen EB. Modulation of short latency stretch reflexes during human hopping.
Acta Physiol Scand 1998b: 163: 181-194.

Wilson GJ, Murphy AJ, Giorgi A. Weight and plyometric training: effects on eccentric and concentric force production. Can J Appl Physiol 1996: 21: 301-315.

Wilson GJ, Newton RU, Murphy AJ, Humphries BJ. The optimal training load for the development of dynamic athletic performance. Med Sci Sports Exerc 1993: 25: 1279-1286.

Zehr EP. Considerations for use of the Hoffmann reflex in exercise studies. Eur J Appl Physiol 2002: 86: 455-468.

Zuur AT, Lundbye-Jensen J, Leukel C, Taube W, Grey MJ, Gollhofer A, Nielsen JB, Gruber M. Contribution of afferent feedback and descending drive to human hopping. J Physiol 2010: 588: 799-807. 